\title{
Do Certain Biomarkers Predict Adverse Outcomes in Coronavirus Disease 2019?
}

\author{
Hiba Narvel $^{\mathrm{a}}$, Anam Sayed ${ }^{\mathrm{b}}$, Nida Narvel ${ }^{\mathrm{b}}$, Shreyas Yakkalia, Tasleem Katchi ${ }^{\mathrm{c}, \mathrm{d}}$
}

\begin{abstract}
Coronavirus disease 2019 (COVID-19) is an infectious disease caused by the severe acute respiratory syndrome coronavirus-2 (SARSCoV-2). Given the rapid spread of the disease, the World Health Organization (WHO) declared the 2019 - 2020 coronavirus outbreak a Public Health Emergency of International Concern (PHEIC) on January 30, 2020, and a pandemic on March 11, 2020. There have been several reports of the limited resources including the lack of intensive care unit (ICU) beds and mechanical ventilators. Thus, biomarkers that predict ICU stay and mortality will be an important tool to appropriately allocate the limited resources. The aim of this review was to identify laboratory markers that can effectively predict the risk of severe infection and increased mortality in COVID-19 cases. We conducted a systematic review of existing literature in six databases to evaluate the predictive value of various biomarkers. We used the keywords "COVID-19", "SARS-CoV-2", "Novel corona virus pneumonia", "Biomarkers", "Adverse outcomes", "Mortality", etc. among many others to refine our search. Several biomarkers were identified to be associated with adverse outcomes in the above studies. These biomarkers can be used as a tool to identify patients at increased risk for adverse outcomes so that the need for aggressive critical care in such patients is met.
\end{abstract}

Keywords: COVID-19; SARS-CoV-2; Novel corona virus pneumonia; Biomarkers; Adverse outcomes; Mortality

\section{Introduction}

Coronavirus disease 2019 (COVID-19) is an infectious disease caused by the severe acute respiratory syndrome coronavirus- 2

Manuscript submitted June 5, 2020, accepted August 22, 2020

Published online April 27, 2021

aSeth GS Medical College and KEM Hospital, Mumbai, India

bIndian Institute of Medical Science and Research, Jalna, India

'Division of Cardiology, HCA-Aventura Hospital and Medical Center, Aventura, FL, USA

${ }^{\mathrm{d} C}$ Corresponding Author: Tasleem Katchi, Division of Cardiology, HCAAventura Hospital and Medical Center, Aventura, FL, USA.

Email: tasleemkatchi@gmail.com

doi: https://doi.org/10.14740/jocmr4254
(SARS-CoV-2) [1]. In comparison to the previous viruses from the corona family, SARS-CoV-2 is not only more contagious but is also spreading more rapidly [2]. Because of its high infectivity, this virus has superseded severe acute respiratory syndrome (SARS) and Middle East respiratory syndrome (MERS) in its death toll. As of June 5, 2020, over 6.7 million cases have been identified worldwide. Of these, over 2.9 million deaths have been reported and over 133,853,038 cases have recovered [3]. The World Health Organization (WHO) declared the 2019 - 2020 coronavirus outbreak a Public Health Emergency of International Concern (PHEIC) $[4,5]$ on January 30, 2020, and a pandemic on March 11, 2020.

The outbreak has cost the world several trillion dollars in terms of government spending and revenue measures to sustain the economy, and additional expenditure on loans, equity injections and guarantees [6].

COVID-19 cases can be classified into asymptomatic, mild, moderate, severe and critical on the basis of symptoms and clinical presentation [7]. Asymptomatic cases refer to carriers of the virus without any manifestations. Mild symptoms include fever, cough, sore throat, malaise, headache, muscle pain, nausea, vomiting, diarrhea, loss of taste and smell, in the absence of shortness of breath, dyspnea, or abnormal chest imaging [7]. These are self-limiting and usually resolve within 2 - 14 days. Individuals with moderate illness show evidence of lower respiratory disease during clinical assessment or imaging and who have an oxygen saturation $\left(\mathrm{SpO}_{2}\right) \geq 94 \%$ on room air at sea level [7]. Severe cases include individuals who have an $\mathrm{SpO}_{2}<94 \%$ on room air at sea level, a ratio of arterial partial pressure of oxygen to fraction of inspired oxygen $\left(\mathrm{PaO}_{2} / \mathrm{FiO}_{2}\right)<300 \mathrm{~mm} \mathrm{Hg}$, respiratory frequency $>$ 30 breaths per minute, or lung infiltrates $>50 \%$ [7]. Individuals who have respiratory failure, septic shock, and/or multiple organ dysfunction are classified as having critical illness [7]. Asymptomatic, mild and most cases of moderate illness may be managed outpatient, whereas severe and critical cases require hospitalization.

There have been several reports of the limited resources including the lack of intensive care unit (ICU) beds and mechanical ventilators $[8,9]$. The healthcare system is overwhelmed by the number and severity of diagnosed cases $[8$, 9]. Thus, biomarkers that predict ICU stay and mortality will be an important tool to appropriately allocate the limited resources. We systematically reviewed and analyzed the published risk factors that have been reported to predict adverse outcomes due to COVID-19. 
We looked at hematological markers, coagulation indices, cardiac biomarkers, inflammatory indices, renal function indices and liver function indices (summarized in Table 1 [10-31]). The aim of this review was to help identify laboratory markers that can effectively predict the risk of severe infection and increased mortality in COVID-19 cases, so that the need for aggressive critical care in such patients is met.

\section{Coagulation Indices}

Han et al compared coagulation parameters in 94 patients admitted to the Renmin Hospital of Wuhan University compared to 40 healthy controls. They reported elevated levels of D-dimer $(10.36 \pm 25.31$ vs. $0.26 \pm 0.18, \mathrm{P}<0.001)$, fibrindegradation products (FDP) $(33.83 \pm 82.28$ vs. $1.55 \pm 1.09)$ and fibrinogen $(5.02 \pm 1.53$ vs. $2.90 \pm 0.53)$ in the COVID-19 group versus the control group [32]. In the above study, patients admitted with COVID-19 were divided into three categories: ordinary, severe and critical. The levels of D-dimer $(2.14 \pm 2.88$ vs. $19.11 \pm 35.48, \mathrm{P}<0.05)$ and FDP $(7.92 \pm 11.38$ vs. $60.01 \pm 108.98, \mathrm{P}<0.05)$ were noted to be significantly elevated in the severe group compared to the ordinary group. However, the prothrombin times (PTs)/international normalized ratio (INR) and partial thromboplastin times were not found to be significantly different among the three groups as well as in comparison to the control group [32].

In a retrospective study of 183 patients admitted to the Tongji Hospital of Huazhong University of Science and Technology in Wuhan with severe COVID-19, the non-survivors were found to have significantly higher D-dimer levels compared to survivors $(2.12 \mathrm{vs} .0 .61 \mu \mathrm{g} / \mathrm{mL}, \mathrm{P}<0.001)$ [33]. The study also reported elevated FDP levels and longer PTs in the non-survivors compared to the survivors on admission ( $\mathrm{P}<$ 0.05 ). Of the patients, $71.4 \%$ of non-survivors and $0.6 \%$ of survivors met the International Society on Thrombosis and Hemostasis diagnostic criteria for disseminated intravascular coagulation (DIC) [33]. The median time from admission to DIC was 4 days (range 1 - 12 days). The proposed mechanism for this derangement is the increased expression of tissue factor and the secretion of von Willebrand factor by activated alveolar macrophages to contain the infection, thereby creating a thrombogenic environment. Zhou et al performed a multivariable regression analysis of 191 patients (54 non-survivors and 137 survivors) and reported that a D-dimer level $>1.0 \mu \mathrm{g} /$ $\mathrm{mL}$ at admission was associated with higher in-hospital mortality (odds ratio (OR): 18.42; confidence interval (CI): 2.64 $128.55 ; \mathrm{P}=0.0033)$ [10]. The development of systemic coagulopathy and the linear relationship between elevated D-dimer and severity of the disease has also been reported in other cases of community-acquired pneumonia (CAP) in previous studies [34-36]. Thus, elevated D-dimer levels may be sensitive for the prognostication of severe COVID-19 infections, but appear to be fairly non-specific.

The coagulopathy seen in severe COVID-19 cases has implications on the use of anticoagulants at prophylactic or therapeutic doses in these patients. Yin et al reported no difference in the 28-day mortality in patients receiving low molecular weight heparin (LMWH) for venous thromboembolism (VTE) prophylaxis compared to those not receiving LMWH. However, when D-dimer levels exceeded $3.0 \mu \mathrm{g} / \mathrm{mL}$, significantly lower mortality was seen in patients receiving LMWH prophylaxis versus those that were not $(32.8 \%$ vs. $52.4 \%, \mathrm{P}=0.017)$ [37]. Moreover, the study by Wang et al revealed significantly elevated D-dimer levels in those who died from novel coronavirus pneumonia compared to those who survived after the pneumonia [38].

\section{Hematological Markers}

Low platelet counts were associated with increased severity of COVID-19 infection [11, 39]. A meta-analysis of nine studies encompassing 1,779 patients conducted by Lippi et al reported an association between thrombocytopenia (defined as a platelet count of less than $150,000 / \mathrm{mm}^{3}$ ) and mortality [11]. In addition, low platelet counts were associated with a five-fold increased risk of mortality in COVID-19 patients (OR: 5.1; 95\% CI: 1.8 - 14.6) [11]. Thus, thrombocytopenia can serve as a clinical indicator of worsening illness in hospitalized patients.

Huang et al conducted a meta-analysis of 3,099 patients from 24 studies and reported that patients with poor outcomes had a lower lymphocyte count (mean difference: $-361.06 \mu \mathrm{L}$ $(-439.18,-282.95), \mathrm{P}<0.001)$ compared to those with good outcome [12]. Guan et al studied the clinical and laboratory characteristics of 1,099 patients admitted with COVID-19 and reported that lymphocytopenia (defined as a lymphocyte count less than $1,500 / \mathrm{mm}^{3}$ ) was present in $83.2 \%$ of the patients [40]. T-lymphocyte counts including CD3, CD4 and CD8 counts were significantly decreased in severe COVID-19 infections, while B-lymphocytes and natural killer cells were not affected [13].

Tan et al retrospectively studied 27 patients admitted to the Hunan Provincial People's Hospital with COVID-19 as well as 75 patients admitted with influenza A or influenza B infections [14]. They categorized COVID-19 patients into four stages: initial stage, progression stage, peak stage and recovery stage, based on chest computed tomography (CT) findings. The median time was 3 days (range 0 - 8 days) in the initial stage, 7 days (range 4 - 12 days) in the progression stage, 12 days (range $8-16$ days) in the peak stage and 16 days (range 11 - 25 days) in the recovery stage after the onset of initial symptoms. Lymphocyte counts decreased progressively in the severe group in the progression and peak stages, followed by an increase in the recovery stage. A similar trend was noted in the granulocyte-lymphocyte ratio which was significantly higher in the severe group in the progression and peak stages but decreased in the recovery stages as the lymphocyte counts recovered.

Platelet-lymphocyte ratio (PLR) is a novel inflammatory index which is a marker of both aggregatory and inflammatory pathways [15]. In a 30-patient case series conducted by Qu et al in the Huizhou Municipal Central Hospital, China, the average PLR of patients with severe infection was 626 [15]. This was significantly higher than the average PLR in non-severe cases, which was $262(\mathrm{P}<0.05)[15] . \Delta \mathrm{PLR}$ is the difference between the PLR at admission and the highest PLR during hospitalization. Qu et al showed that $\triangle \mathrm{PLR}$ correlated with the 


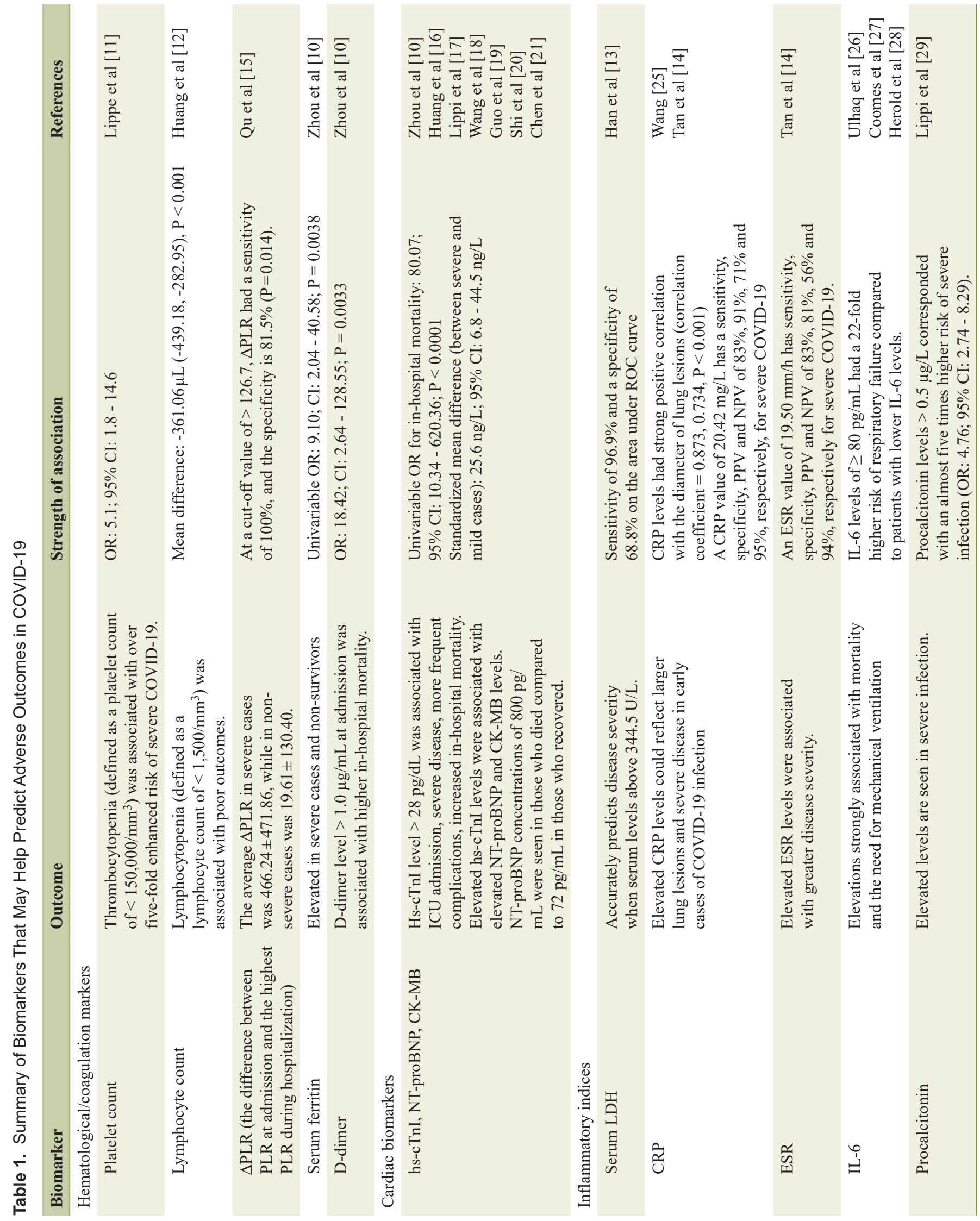




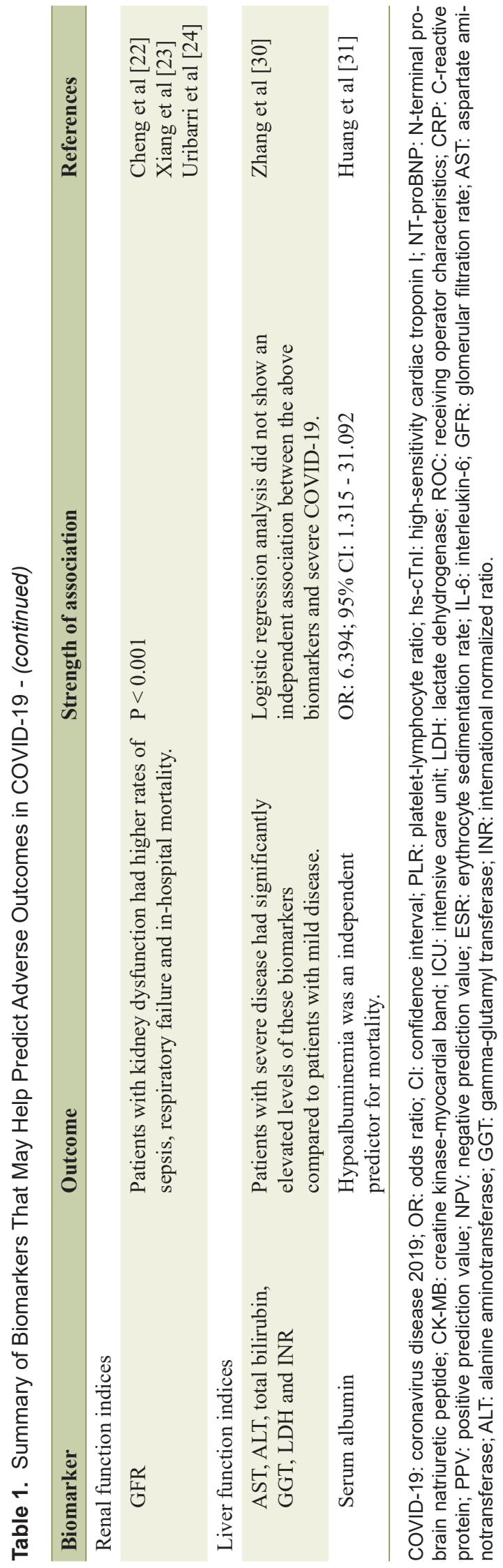

length of stay [15]. In the above study, the average $\triangle P L R$ in severe cases was $466.24 \pm 471.86$, while in non-severe cases was $19.61 \pm 130.40[12,14,15]$. An increase in $\triangle P L R$ during treatment was associated with longer hospitalization and greater severity of infection [15]. The study identified that, at a cut-off value of $>126.7, \triangle$ PLR had a sensitivity of $100 \%$, the specificity is $81.5 \%(\mathrm{P}=0.014)$ and levels greater than this value suggested a need for more aggressive intervention. However, the study is limited by its small sample size [15]. Platelet counts seem to increase in the initial stages of infection likely secondary to reactive thrombocytosis. However, decreased counts are seen as the infection progressed reflective of bone marrow suppression secondary to inflammatory cytokines. Platelet counts correlated positively with the length of hospital stay whereas lymphocyte count correlated negatively [15].

Zhou et al reported that the odds of in-hospital mortality were higher in patients with elevated serum ferritin levels ( $>$ $300 \mu \mathrm{g} / \mathrm{L}$ ) (univariable OR: 9.10; CI: 2.04 - 40.58; P=0.0038) [10]. The proposed mechanism for higher ferritin levels is the key pathogenic molecular step of SARS-CoV-2 which involves the lysis of the beta-2 chain of the hemoglobin molecule, followed by the dissociation and release of free iron into the circulation [41]. This impairs the oxygen-carrying capacity of hemoglobin leading to hypoxia and multi-organ failure [41]. The free iron is toxic to alveolar macrophages, causing the typical ground-glass opacities on computed tomography. The increased ferritin levels lead to increased macrophage production. This, in turn, leads to lymphocytopenia due to this preferential differentiation into monocytes over lymphocytes. The increased iron load and a compensatory increase in hemoglobin lead to increased blood viscosity and increased risk of thrombosis, which may be the reason for the elevated D-dimer levels seen in severe cases.

\section{Cardiac Biomarkers}

Huang et al reported myocardial injury in five of the first 41 patients diagnosed with COVID-19 in Wuhan, evidenced by elevated high-sensitivity cardiac troponin I (hs-cTnI) levels (above the 99th percentile of the upper reference limit (URL), $>28 \mathrm{pg} / \mathrm{mL}$ ) [16]. Of these five patients, four required ICU admission. Lippi et al conducted a meta-analysis of four studies with a total of 341 patients looking at the association between cardiac troponin and COVID-19 [17]. They found that the values of cTnI were significantly higher in patients with severe COVID-19 than in those with non-severe disease (standardized mean difference, $25.6 \mathrm{ng} / \mathrm{L} ; 95 \%$ CI: 6.8 - $44.5 \mathrm{ng} / \mathrm{L}$ ) [17]. In a study of 138 hospitalized patients with COVID-19 in Wuhan, China, myocardial injury as evidenced by elevated hs-cTnI or new electrocardiographic or echocardiographic abnormalities, was present in $7.2 \%$ of patients overall, but in as many as $22 \%$ patients that required ICU care [18]. In a retrospective study of 191 patients with laboratory-confirmed COVID-19 from Jinyintan Hospital and Wuhan Pulmonary Hospitals, elevated hscTnI level $(>28 \mathrm{pg} / \mathrm{mL})$ was reported in $4.1 \%$ of all patients, $3 \%$ of survivors, but in as many as one-fifth $(22.1 \%)$ of nonsurvivors $(\mathrm{P}<0.001)$. Elevated hs-cTnI level was found to be a predictor of in-hospital death (univariable OR: $80.07,95 \% \mathrm{CI}$ : 
10.34 - 620.36; $\mathrm{P}<0.0001)$ [10]. A rapid increase in cTnI was noted beyond day 16 after the onset of disease in non-survivors (from $24.7 \mathrm{pg} / \mathrm{mL}$ on day 7 to $55.7 \mathrm{pg} / \mathrm{mL}$ on day 13, to 134.5 $\mathrm{pg} / \mathrm{mL}$ on day 19 and to $290.6 \mathrm{pg} / \mathrm{mL}$ on day 22 ).

The National Health Commission of China reported that elevated troponin levels or in-hospital cardiac arrest were seen in almost $12 \%$ of patients without known cardiovascular diseases [42]. Elevated troponins were associated with increased mortality in both patients with and without underlying cardiovascular diseases $[43,44]$. Guo et al conducted a study of 187 patients admitted with COVID-19 to the seventh Hospital of Wuhan City, China [19]. They reported that elevated TnT levels were more likely to be seen in patients with underlying cardiovascular disease $(54.5 \%)$ compared with patients without underlying cardiovascular disease (13.2\%) [19]. Patients with elevated TnT levels developed more frequent complications, including acute respiratory distress syndrome $(57.7 \%$ vs. $11.9 \%)$, malignant arrhythmias (11.5\% vs. $5.2 \%)$ including ventricular tachycardia/ventricular fibrillation, acute coagulopathy (65.8\% vs. $20.0 \%)$ and acute kidney injury (36.8 vs. $4.7 \%$ ), compared with those with normal TnT levels. The study reported markedly higher mortality in patients with elevated plasma TnT levels $(59.6 \%)$ than in patients with normal $(8.9 \%)$ levels [19]. Elevated troponins were associated with increased mortality in both, patients with underlying cardiovascular diseases (69.44\%) and without (37.50\%). Mortality in patients with underlying cardiovascular diseases but normal troponin was $13.33 \%$ [19].

Shi at al performed a retrospective cohort study of 416 consecutive patients admitted to the Renmin Hospital of Wuhan University with laboratory-confirmed COVID-19 and reported results similar to those reported by Guo et al. Patients with cardiac injury (hs-TnI above the 99th percentile URL) were older and more likely to have comorbidities such as hypertension, diabetes, congestive heart failure and chronic obstructive pulmonary disease [20]. Elevated cTnI levels were associated with elevated levels of other biomarkers of cardiac injury as well, specifically creatine kinase-myocardial band (CK-MB) test (median (interquartile range (IQR)): 3.34 (2.11 - 5.80) vs. $0.81(0.54$ - 1.38$) \mathrm{ng} / \mathrm{mL})$ and $\mathrm{N}$-terminal pro-brain natriuretic peptide (NT-proBNP) (median (IQR): 817.4 (336.0 - 1,944.0) vs. $141.4(39.3$ - 303.6) pg/mL), as well as higher levels of lactic acid (median (IQR): 2.10 (1.40 - 3.10) vs. 1.80 (1.30 - 2.10) $\mathrm{mm} \mathrm{Hg}$ ), higher levels of creatinine (median (IQR): $0.79(0.71-1.17)$ vs. $0.63(0.55-0.79) \mathrm{mg} / \mathrm{dL})$ and aspartate aminotransferase (AST) (median (IQR): 39.5 (27.2 - 57.8) vs. $29.0(21.0$ - 39.0) U/L) [19]. Shi et al reported that patients with cardiac injury showed higher median leukocyte count (median (IQR): 9,400 (6,900 - 13,800) cells/ $\mu$ L vs. 5,500 $(4,200-7,400)$ cells $/ \mu \mathrm{L})$, higher levels of high-sensitivity Creactive protein (hs-CRP) (median (IQR): 10.2 (6.4 - 17.0) mg/ dL vs. $3.7(1.0$ - 7.3) mg/dL), procalcitonin (median (IQR): $0.27(0.10$ - 1.22) ng/mL vs. $0.06(0.03-0.10) \mathrm{ng} / \mathrm{mL})$, CKMB (median (IQR): $3.2(1.8$ - 6.2) ng/mL vs. $0.9(0.6-1.3) \mathrm{ng} /$ $\mathrm{mL}$ ), myohemoglobin (median (IQR): 128 (68 - 305) $\mu \mathrm{g} / \mathrm{L}$ vs. $39(27$ - 65) $\mu \mathrm{g} / \mathrm{L})$, NT-proBNP (median (IQR): 1,689 (698 3,327) pg/mL vs. 139 (51 - 335) pg/mL), AST (median (IQR): $40(27-60) \mathrm{U} / \mathrm{L}$ vs. $29(21-40) \mathrm{U} / \mathrm{L})$ and creatinine (median (IQR): 1.15 (0.72 - 1.92) mg/dL vs. $0.64(0.54-0.78) \mathrm{mg} / \mathrm{dL})$ during hospitalization [20].

Chen et al reported a median hs-cTnI level of $40.8 \mathrm{ng} / \mathrm{L}$ (URL $15.6 \mathrm{ng} / \mathrm{L}$ ) in patients who died compared to $3.3 \mathrm{ng} / \mathrm{L}$ in those who survived, as well as mean NT-proBNP concentrations of $800 \mathrm{pg} / \mathrm{mL}$ in those who died compared to $72 \mathrm{pg} / \mathrm{mL}$ in patients who recovered [21]. Both Guo et al and Shi et al reported that plasma TnT levels demonstrated a positive linear correlation with plasma high-sensitivity C-reactive protein (hs-CRP) levels. Hs-CRP is a marker of inflammation and NTproBNP is a marker of ventricular dysfunction. The above association suggests that cardiac injury, as evidenced by elevated troponin levels, was associated with greater severity of inflammation and ventricular dysfunction in COVID-19 patients.

SARS-CoV-2 has a spike protein receptor-binding domain, similar to SARS-CoV-1, which interacts with the angiotensin-converting enzyme-2 (ACE-2) receptors [45]. ACE-2 receptors are present in lung alveoli, enterocytes, vascular endothelial cells, kidneys and heart [46]. The presence of ACE2 receptors on myocytes may be the mechanism behind the frequent myocardial inflammation in COVID-19 cases. The ACE-2 receptor is upregulated in patients with cardiovascular disease, especially with the use of the renin-angiotensinaldosterone system inhibitors [42], which led to the speculation about the potential effects of ACE inhibitors (ACEIs) or angiotensin-receptor blockers (ARBs) on COVID-19 patients. However, Guo et al demonstrated no significant difference in the mortality rates of patients with and without the use of ACEIs/ARBs (36.8\% vs. 21.4\%; $\mathrm{P}=0.13)$ [19].

Sandoval et al suggest that cTn elevations in COVID-19 patients should be broadly classified as either: 1) chronic myocardial injury $(<20 \%$ change cTn levels, may be seen in many COVID-19 patients because of the high prevalence of chronic cardiovascular conditions); 2) acute non-ischemic myocardial injury (may be seen in cases of myocarditis, myopericarditis, acute heart failure, pulmonary embolism and stress cardiomyopathy); or 3) acute myocardial infarction (MI) (type 1 MI due to atherosclerotic plaque rupture or coronary thrombosis, and type $2 \mathrm{MI}$ due to supply-demand mismatch driven by hypoxia, hypotension, acidosis, etc.) [47].

Serial troponin measurements as opposed to single-point measurements on admission can better identify patients with progression towards a worse clinical picture who may need aggressive critical care. Serial troponin measurements may also help identify patients that may require additional imaging such as echocardiography. In order to minimize exposure to the healthcare staff, focused imaging may be performed in COVID-19 patients with normal or modestly elevated levels of cTn, BNP and normal electrocardiogram, whereas detailed imaging studies may be performed in patients with dramatic increases in cardiac biomarker levels, particularly if accompanied by hemodynamic instability, unequivocal ischemic symptoms, electrocardiographic abnormalities, or left ventricular dysfunction on point of care ultrasound. Imaging studies should only be performed if they are believed to impact management.

Patients with COVID-19 may present with ST-segment elevation in the absence of obstructive coronary artery disease. In patients with a high clinical suspicion for ST-elevation MI, percutaneous coronary intervention (PCI) remains the standard of care at PCI-capable hospitals when it can be provided 
in a timely fashion, with an expert care team outfitted with personal protection equipment and in a dedicated cardiac catheterization laboratory, according to the statement published on April 20, 2020 [48].

\section{Renal Function Indices}

Cheng et al conducted the first prospective cohort study of adult patients with COVID-19, cases with elevated serum creatinine levels had significantly higher mortality of $33.7 \%$ compared with a mortality of $13.2 \%$ in cases with normal serum creatinine levels [22]. In the above study, after adjusting for age, sex, comorbidities, disease severity and lymphocyte count, Kaplan-Meir analysis revealed that the following biomarkers were all associated with in-hospital death: proteinuria of any degree, hematuria of any degree, elevated blood urea nitrogen $(\mathrm{BUN})$, serum creatinine, peak serum creatinine $>133$ $\mu \mathrm{mol} / \mathrm{L}$ and acute kidney injury (AKI) beyond stage 2 [22]. They concluded that the presence of kidney disease and the development of AKI during hospitalization were associated with higher in-hospital mortality. Xiang et al studied 28 patients who were diagnosed with COVID-19 at the First Affiliated Hospital of Zunyi Medical University and The Fourth People's Hospital of Zunyi city and reported that serum urea, creatinine and cystatin $\mathrm{C}$ levels, which reflect glomerular filtration rate (GFR) were significantly higher in severe COVID-19 cases compared to mild cases [23].

The mechanism of kidney injury in COVID-19 is unclear and may involve direct cytopathic effects of the virus that gains access to the cells through ACE- 2 receptors, or the indirect effects of cytokines. Deposition of immune complexes could be another mechanism of kidney injury; however, kidney specimens from patients infected with SARS did not show evidence of immune-mediated glomerular damage [22]. In an analysis of the health outcome predictive evaluation for COVID-19 (HOPE COVID-19) registry, patients with kidney dysfunction had higher rates of sepsis (estimated GFR (eGFR) $>60$ vs. eGFR 30 - 60 vs. eGFR $<30,1.9 \%$ vs. $26.4 \%$ vs. $40.8 \%$, $\mathrm{P}<0.001$ ), respiratory failure (eGFR $>60$ vs. eGFR 30 - 60 vs. eGFR $<30,35.4 \%$ vs. $72.2 \%$ vs. $62.0 \%, \mathrm{P}<0.001)$ and in-hospital mortality [24]. Patients with eGFR $>60 \mathrm{~mL} / \mathrm{min} / 1.73 \mathrm{~m}^{2}$ were reported to have an in-hospital mortality of $18.4 \%$ versus $56.5 \%$ in patients with eGFR $30-60 \mathrm{~mL} / \mathrm{min} / 1.73 \mathrm{~m}^{2}$ and $65.5 \%$ in patients with eGFR $<30 \mathrm{~mL} / \mathrm{min} / 1.73 \mathrm{~m}^{2}(\mathrm{P}<0.001)$ [24].

\section{Inflammatory Indices}

Han et al conducted a study of 107 patients admitted with COVID-19 to the Renmin Hospital of China. They reported that lactate dehydrogenase (LDH) levels correlated positively with various disease severity scores such as the acute physiology and chronic health evaluation (APACHE), the sequential organ failure assessment (SOFA) scores, the pneumonia severity index (PSI) and the confusion/urea/respiratory rate/blood pressure (CURB)-65 scores [13]. They reported that the severe cases had a higher APACHE score (8.5 vs. 4.0, $\mathrm{P}<0.001)$ and a higher SOFA score (2 vs. $1, \mathrm{P}<0.001)$ compared to nonsevere cases on admission as well as a higher PSI score $(83.35$ vs. 55.76, $\mathrm{P}<0.001)$ and a CURB-65 score ( 1 vs. $0, \mathrm{P}<0.001)$ [13]. On a Pearson correlation analysis, a positive correlation was obtained between LDH levels and the APACHE, SOFA and PSI scores. The area under the receiving operator characteristics (ROC) curve also revealed that serum LDH levels above $344.5 \mathrm{U} / \mathrm{L}$ can be a predictor of severe infection with a sensitivity of $96.9 \%$ and a specificity of $68.8 \%$ [13]. In this study, LDH levels correlated positively with CRP levels, and CRP levels above $88.5 \mathrm{mg} / \mathrm{L}$ were reported to have moderate accuracy in predicting severe disease. Several studies have reported elevated CRP levels in severe cases [25, 49]. Wang studied the correlation between CRP levels and the diameter of lung lesions seen on chest CT [25]. CRP levels and the diameter of the largest lung lesion in the moderate group were higher than those in the mild group (Mann-Whitney test = $-2.647,-2.171, \mathrm{P}<0.05)$, those in the severe group were higher than those in the moderate group (Mann-Whitney test $=0.693$, $-2.177, \mathrm{P}<0.05)$ and those in the critical group were higher than those in the severe group (Mann-Whitney test $=-0.068$, $-1.549, \mathrm{P}<0.05$ ) [25]. Hence, elevated CRP levels could reflect larger lung lesions and severe disease in early cases of COVID-19 infection. Xiang et al reported that elevated CRP levels were present in most cases of COVID-19 infection, but the levels were not significantly different in mild and severe cases [23].

Tan et al reported that CRP levels were significantly higher in patients with severe COVID-19 compared to mild cases in the initial stage; however, a significant difference in CRP levels between the two groups was not seen in the later stages [14]. In this study, rising CRP levels were seen independent of worsening computed tomography (CT) findings, suggesting that CRP may be an early predictor of worsening infection [14]. Erythrocyte sedimentation rate (ESR) levels were found to be significantly elevated in the severe group in the initial stage, but not in the progression, peak and recovery stages [14]. They reported that, at a cut-off value of $20.42 \mathrm{mg} / \mathrm{L}$, the sensitivity, specificity, positive prediction value (PPV) and negative prediction value (NPV) of CRP were 83\%, 91\%, 71\% and $95 \%$, respectively [14]. At a cut-off value of $19.50 \mathrm{~mm} / \mathrm{h}$, the sensitivity, specificity, PPV and NPV of ESR were $83 \%$, $81 \%, 56 \%$ and $94 \%$, respectively. The PPV of CRP to predict the severity of COVID-19 appeared to be superior to that of chest CT findings [14].

A meta-analysis of nine studies by Ulhaq et al demonstrated elevated iinterleukin-6 (IL-6) levels in severe cases on COVID-19 compared to non-severe cases [26]. Another metaanalysis of six studies $(n=1,302)$ revealed a 2.9 -fold elevation in IL-6 in severe cases of COVID-19 infection compared to non-severe cases [27]. Herold et al reported that higher IL-6 levels were strongly associated with respiratory failure and the need for mechanical ventilation in COVID-19 patients $(\mathrm{P}=$ 0.00001 ) [28]. Patients with IL-6 levels of $\geq 80 \mathrm{pg} / \mathrm{mL}$ had a 22 -fold higher risk of respiratory failure compared to patients with lower IL-6 levels. Tocilizumab is an anti-IL-6 receptor antibody. Guaraldi et al conducted a retrospective cohort study to assess the role of tocilizumab in reducing the risk for in- 
vasive mechanical ventilation and death in patients admitted with severe COVID-19 and receiving standard of care in Italy [50]. They reported that after adjusting for sex, age, recruiting center, duration of symptoms and SOFA score, tocilizumab treatment was associated with a reduced risk of invasive mechanical ventilation or death (adjusted hazard ratio: $0.61 ; 95 \%$ CI: $0.40-0.92 ; \mathrm{P}=0.020$ ) [50]. The COVACTA trial was designed to evaluate the efficacy, safety, pharmacodynamics and pharmacokinetics of tocilizumab compared with a matching placebo in combination with standard of care in hospitalized patients with severe COVID-19 pneumonia [51]. The results of the trial were released in July 2020; the trial did not meet its primary endpoint of improved clinical status in patients receiving tocilizumab versus placebo, or the secondary endpoint of improved mortality [51]. However, a positive trend in time to hospital discharge was noted in patients receiving tocilizumab [51].

Another biomarker with the potential to predict severity is procalcitonin $[16,29]$. Huang et al reported elevated procalcitonin levels in $25 \%$ of patients who were admitted to the ICU compared with $0 \%$ who were not $(\mathrm{P}=0.029)$ [16]. A metaanalysis by Lippi et al reported that procalcitonin levels $>0.5$ $\mu \mathrm{g} / \mathrm{L}$ corresponded with an almost five times higher risk of severe infection (OR: 4.76; 95\% CI: 2.74 - 8.29) [29].

\section{Liver Function Indices}

Zhang et al conducted a retrospective study of 115 patients admitted with COVID-19 using 114 patients admitted with CAP as a control group [30]. Within the COVID-19 group, patients with severe disease had significantly elevated levels of serum aspartate aminotransferase (AST), alanine aminotransferase (ALT), total bilirubin, gamma-glutamyl transferase (GGT), LDH and INR levels compared to patients with mild disease [30]. Albumin levels were significantly lower in severe COVID-19 cases, and globulin levels were significantly higher, compared to mild cases [30]. There was no significant difference in the alkaline phosphatase (ALP) levels between the severe and mild groups [30]. However, in this study, logistic regression analysis did not show an independent association between the above biomarkers and severe COVID-19 [30]. Wu et al conducted a meta-analysis of 45 studies to assess the association between abnormal liver biomarkers and the severity of COVID-19 [52]. The meta-analysis found that the levels of AST and ALT levels were significantly higher, while albumin levels were significantly lower in the severe and critical groups compared to the mild and moderate groups [52]. Huang et al reported that hypoalbuminemia predicts the outcome of $\mathrm{COV}$ ID-19 independent of age and co-morbidity [31].

\section{Comparison With Other Forms of CAP}

The above studies indicate that several biomarkers can help predict adverse outcomes in COVID-19. The sensitivity of these biomarkers in predicting mortality and length of ICU/hospital stay appears questionable; however, the specificity is poor as several biomarkers are elevated in other infectious processes as well. COVID-19 may mimic influenza pneumonia in the early stages and it becomes important to differentiate between the two to ensure adequate isolation and supportive care. In the study by Tan et al, significantly higher levels of granulocytes and monocytes were seen in patients admitted with influenza, compared to patients admitted with COVID-19; however, no significant difference was seen in the total leucocyte count, lymphocytes, hemoglobin, hematocrit and platelet counts, which further validates the poor specificity of these biomarkers [24]. As previously stated, the linear relationship between elevated D-dimer levels and severity has been demonstrated in other cases of CAP as well; however, a mortality benefit with the use of LMWH for VTE prophylaxis was only seen in COVID-19 patients with D-dimer levels exceeding $3 \mu \mathrm{g} / \mathrm{mL}$, and not in patients admitted with other forms of CAP.

\section{Conclusion}

Several biomarkers have been identified to be associated with adverse outcomes in various studies. The sensitivity of these biomarkers is questionable and specificity appears to be poor. Nevertheless, these biomarkers can be used as a tool to identify patients at increased risk for adverse outcomes, to ensure appropriate up-triaging of patients and appropriate allocation of limited resources. We believe that the research on predictive biomarkers is in the early stages and sincerely urge clinicians across the world to contribute further to this research.

\section{Acknowledgments}

We are indebted to our families for their unconditional support.

\section{Financial Disclosure}

None to declare.

\section{Conflict of Interest}

None to declare.

\section{Author Contributions}

HN, SY, and TK designed the study. HN, SY, and TK reviewed the relevant literature. $\mathrm{HN}$ and TK drafted the manuscript. All authors contributed to manuscript revisions and approved the final manuscript.

\section{Data Availability}

Any inquiries regarding supporting data availability of this study should be directed to the corresponding author. 


\section{References}

1. Yuen KS, Ye ZW, Fung SY, Chan CP, Jin DY. SARSCoV-2 and COVID-19: the most important research questions. Cell Biosci. 2020;10:40.

2. Walls AC, Park YJ, Tortorici MA, Wall A, McGuire AT, Veesler D. Structure, function, and antigenicity of the SARS-CoV-2 spike glycoprotein. Cell. 2020;181(2):281292 e286.

3. Worldometer. Coronavirus Cases. https://www.worldometers.info/coronavirus/coronavirus-cases/\#daily-cases.

4. Hundreds of evacuees to be held on bases in California; Hong Kong and Taiwan restrict travel from mainland China - The Washington Post. https://www. washingtonpost.com/world/asia_pacific/coronavirus-china-live-updates $/ 2020 / 02 / 05 / 114$ ced8a-479c-11ea-bc 78 8a18f7afcee7_story.html.

5. Statement on the second meeting of the International Health Regulations. 2005. Emergency Committee regarding the outbreak of novel coronavirus. https://www. who.int/news-room/detail/30-01-2020-statement-on-thesecond-meeting-of-the-international-health-regulations(2005)-emergency-committee-regarding-the-outbreakof-novel-coronavirus-(2019-ncov).

6. Global Financial Stability Report. https://www.imf.org/ en/Publications/GFSR/Issues/2020/04/14/Global-Financial-Stability-Report-April-2020-49020.

7. ClinicalPresentationofPeoplewithSARS-CoV-2Infection. https://www.covid19treatmentguidelines.nih.gov/over view/clinical-presentation/.

8. Xie J, Tong Z, Guan X, Du B, Qiu H, Slutsky AS. Critical care crisis and some recommendations during the COVID-19 epidemic in China. Intensive Care Med. 2020;46(5):837-840.

9. Emanuel EJ, Persad G, Upshur R, Thome B, Parker M, Glickman A, Zhang C, et al. Fair allocation of scarce medical resources in the time of Covid-19. N Engl J Med. 2020;382(21):2049-2055.

10. Zhou F, Yu T, Du R, Fan G, Liu Y, Liu Z, Xiang J, et al. Clinical course and risk factors for mortality of adult inpatients with COVID-19 in Wuhan, China: a retrospective cohort study. Lancet. 2020;395(10229):1054-1062.

11. Lippi G, Plebani M, Henry BM. Thrombocytopenia is associated with severe coronavirus disease 2019 (COVID-19) infections: A meta-analysis. Clin Chim Acta. 2020;506:145-148.

12. Huang I, Pranata R. Lymphopenia in severe coronavirus disease-2019 (COVID-19): systematic review and metaanalysis. J Intensive Care. 2020;8:36.

13. Han Y, Zhang H, Mu S, Wei W, Jin C, Tong C, Song Z, et al. Lactate dehydrogenase, an independent risk factor of severe COVID-19 patients: a retrospective and observational study. Aging (Albany NY). 2020;12(12):1124511258.

14. Tan C, Huang Y, Shi F, Tan K, Ma Q, Chen Y, Jiang X, et al. C-reactive protein correlates with computed tomographic findings and predicts severe COVID-19 early. J Med Virol. 2020;92(7):856-862.
15. Qu R, Ling Y, Zhang YH, Wei LY, Chen X, Li XM, Liu $\mathrm{XY}$, et al. Platelet-to-lymphocyte ratio is associated with prognosis in patients with coronavirus disease-19. J Med Virol. 2020;92(9):1533-1541.

16. Huang C, Wang Y, Li X, Ren L, Zhao J, Hu Y, Zhang $\mathrm{L}$, et al. Clinical features of patients infected with 2019 novel coronavirus in Wuhan, China. Lancet. 2020;395(10223):497-506.

17. Lippi G, Lavie CJ, Sanchis-Gomar F. Cardiac troponin I in patients with coronavirus disease 2019 (COVID-19): Evidence from a meta-analysis. Prog Cardiovasc Dis. 2020;63(3):390-391.

18. Wang D, Hu B, Hu C, Zhu F, Liu X, Zhang J, Wang B, et al. Clinical characteristics of 138 hospitalized patients with 2019 novel coronavirus-infected pneumonia in $\mathrm{Wu}-$ han, China. JAMA. 2020;323(11):1061-1069.

19. Guo T, Fan Y, Chen M, Wu X, Zhang L, He T, Wang H, et al. Cardiovascular implications of fatal outcomes of patients with coronavirus disease 2019 (COVID-19). JAMA Cardiol. 2020;5(7):811-818.

20. Shi S, Qin M, Shen B, Cai Y, Liu T, Yang F, Gong W, et al. Association of cardiac injury with mortality in hospitalized patients with COVID-19 in Wuhan, China. JAMA Cardiol. 2020;5(7):802-810.

21. Chen T, Wu D, Chen H, Yan W, Yang D, Chen G, Ma $\mathrm{K}$, et al. Clinical characteristics of 113 deceased patients with coronavirus disease 2019: retrospective study. BMJ. 2020;368:m1091.

22. Cheng Y, Luo R, Wang K, Zhang M, Wang Z, Dong L, Li $\mathrm{J}$, et al. Kidney disease is associated with in-hospital death of patients with COVID-19. Kidney Int. 2020;97(5):829838.

23. Xiang J, Wen J, Yuan X, Xiong S, Zhou X, Liu C, et al. Potential biochemical markers to identify severe cases among COVID-19 patients. MedRxiv preprint.

24. Uribarri A, Nunez-Gil IJ, Aparisi A, Becerra-Munoz VM, Feltes G, Trabattoni D, Fernandez-Rozas I, et al. Impact of renal function on admission in COVID-19 patients: an analysis of the international HOPE COVID-19 (Health Outcome Predictive Evaluation for COVID 19) Registry. J Nephrol. 2020;33(4):737-745.

25. Wang L. C-reactive protein levels in the early stage of COVID-19. Med Mal Infect. 2020;50(4):332-334.

26. Ulhaq ZS, Soraya GV. Interleukin-6 as a potential biomarker of COVID-19 progression. Med Mal Infect. 2020;50(4):382-383.

27. Coomes EA, Haghbayan H. Interleukin-6 in Covid-19: A systematic review and meta-analysis. Rev Med Virol. 2020;30(6):1-9.

28. Herold T, Jurinovic V, Arnreich C, Hellmuth JC, Bergwelt-Baildon M, Klein M, et al. Level of IL-6 predicts respiratory failure in hospitalized symptomatic COVID-19 patients. MedRxiv. 2020;20047381.

29. Lippi G, Plebani M. Procalcitonin in patients with severe coronavirus disease 2019 (COVID-19): A meta-analysis. Clin Chim Acta. 2020;505:190-191.

30. Zhang Y, Zheng L, Liu L, Zhao M, Xiao J, Zhao Q. Liver impairment in COVID-19 patients: A retrospective analysis of 115 cases from a single centre in Wuhan city, China. 
Liver Int. 2020;40(9):2095-2103.

31. Huang J, Cheng A, Kumar R, Fang Y, Chen G, Zhu Y, Lin S. Hypoalbuminemia predicts the outcome of COVID-19 independent of age and co-morbidity. J Med Virol. 2020;92(10):2152-2158.

32. Han H, Yang L, Liu R, Liu F, Wu KL, Li J, Liu XH, et al. Prominent changes in blood coagulation of patients with SARS-CoV-2 infection. Clin Chem Lab Med. 2020;58(7):1116-1120.

33. Tang N, Li D, Wang X, Sun Z. Abnormal coagulation parameters are associated with poor prognosis in patients with novel coronavirus pneumonia. J Thromb Haemost. 2020;18(4):844-847.

34. Shilon Y, Shitrit AB, Rudensky B, Yinnon AM, Margalit M, Sulkes J, Shitrit D. A rapid quantitative D-dimer assay at admission correlates with the severity of community acquired pneumonia. Blood Coagul Fibrinolysis. 2003;14(8):745-748.

35. Abraham E. Coagulation abnormalities in acute lung injury and sepsis. Am J Respir Cell Mol Biol. 2000;22(4):401404.

36. Rijneveld AW, Weijer S, Bresser P, Florquin S, Vlasuk GP, Rote WE, Spek CA, et al. Local activation of the tissue factor-factor VIIa pathway in patients with pneumonia and the effect of inhibition of this pathway in murine pneumococcal pneumonia. Crit Care Med. 2006;34(6):1725-1730.

37. Yin S, Huang M, Li D, Tang N. Difference of coagulation features between severe pneumonia induced by SARSCoV2 and non-SARS-CoV2. J Thromb Thrombolysis. 2020.

38. Wang L, He W, Yu X, Hu D, Bao M, Liu H, Zhou J, et al. Coronavirus disease 2019 in elderly patients: Characteristics and prognostic factors based on 4-week follow-up. J Infect. 2020;80(6):639-645.

39. Liu Y, Sun W, Guo Y, Chen L, Zhang L, Zhao S, Long D, et al. Association between platelet parameters and mortality in coronavirus disease 2019: Retrospective cohort study. Platelets. 2020;31(4):490-496.

40. Guan WJ, Ni ZY, Hu Y, Liang WH, Ou CQ, He JX, Liu $\mathrm{L}$, et al. Clinical Characteristics of Coronavirus Disease 2019 in China. N Engl J Med. 2020;382(18):1708-1720.

41. Liu W, Li H. COVID-19: Attacks the 1-beta chain of hemoglobin and captures the porphyrin to inhibit human heme metabolism. https://chemrxiv.org/ndownloader/ files/22283226.

42. Zheng YY, Ma YT, Zhang JY, Xie X. COVID-19 and the cardiovascular system. Nat Rev Cardiol. 2020;17(5):259260.

43. Ruan Q, Yang K, Wang W, Jiang L, Song J. Clinical predictors of mortality due to COVID-19 based on an analysis of data of 150 patients from Wuhan, China. Intensive Care Med. 2020;46(5):846-848.

44. Tersalvi G, Vicenzi M, Calabretta D, Biasco L, Pedrazzini G, Winterton D. Elevated troponin in patients with coronavirus disease 2019: possible mechanisms. J Card Fail. 2020;26(6):470-475.

45. Rali AS, Ranka S, Shah Z, Sauer AJ. Mechanisms of myocardial injury in coronavirus disease 2019. Card Fail Rev. 2020;6:e15.

46. Luan J, Lu Y, Jin X, Zhang L. Spike protein recognition of mammalian ACE2 predicts the host range and an optimized ACE2 for SARS-CoV-2 infection. Biochem Biophys Res Commun. 2020;526(1):165-169.

47. Sandoval Y, Januzzi JL, Jr., Jaffe AS. Cardiac troponin for assessment of myocardial injury in COVID-19: JACC review topic of the week. J Am Coll Cardiol. 2020;76(10):1244-1258.

48. ACC, SCAI, ACEP release consensus statement on management of AMI patients during COVID-19.

49. Chen C, Chen C, Yan JT, Zhou N, Zhao JP, Wang DW. [Analysis of myocardial injury in patients with COVID-19 and association between concomitant cardiovascular diseases and severity of COVID-19]. Zhonghua Xin Xue Guan Bing Za Zhi. 2020;48(7):567-571.

50. Guaraldi G, Meschiari M, Cozzi-Lepri A, Milic J, Tonelli R, Menozzi M, Franceschini E, et al. Tocilizumab in patients with severe COVID-19: a retrospective cohort study. Lancet Rheumatol. 2020;2(8):e474-e484.

51. Genetech provides an update on the Phase III COVACTA trial of Actemra in hospitalized patients with severe COVID-19 associated pneumonia. Genentech. July 28, 2020.

52. Wu Y, Li H, Guo X, Yoshida EM, Mendez-Sanchez N, Levi Sandri GB, Teschke R, et al. Incidence, risk factors, and prognosis of abnormal liver biochemical tests in COVID-19 patients: a systematic review and meta-analysis. Hepatol Int. 2020;14(5):621-637. 